\title{
Impact of Cerebrovascular Disease on Survival Benefits from Local Treatment in Patients with De Novo Metastatic Hormone-Sensitive Prostate Cancer
}

\author{
Tae Jin Kim ${ }^{1}$, Young Dong Yu ${ }^{1}$, Dong Soo Park ${ }^{1}$, Koon Ho Rha ${ }^{2}$, Sung Joon Hong ${ }^{2}$, \\ Kang Su Cho ${ }^{3}$, Byung Ha Chung, and Kyo Chul Koo ${ }^{3}$ \\ ${ }^{1}$ Department of Urology, CHA Bundang Medical Center, CHA University School of Medicine, Seongnam; \\ ${ }^{2}$ Department of Urology, Severance Hospital, Yonsei University College of Medicine, Seoul; \\ ${ }^{3}$ Department of Urology, Gangnam Severance Hospital, Yonsei University College of Medicine, Seoul, Korea.
}

Purpose: Local treatment has become a treatment option for patients with de novo metastatic hormone-sensitive prostate cancer (mHSPC). Subgroup analyses based on a history of cerebrovascular disease (CVD) were performed to evaluate the impact thereof on overall survival (OS) after local treatment.

Materials and Methods: A retrospective analysis was performed for 879 patients with de novo mHSPC between August 2003 and November 2016. Patients were stratified according to prior CVD history and the type of initial treatment: androgen-deprivation therapy (ADT) alone versus local treatment consisting of radical prostatectomy (RP) or radiation therapy (RT) with ADT, with or without metastasis-directed therapy. The primary outcome was OS assessed by Kaplan-Meier analysis and Cox-regression models.

Results: Of 879 patients, 660 (75.1\%) men underwent ADT alone, and 219 (24.9\%) men underwent RP or RT with ADT, with or without metastasis-directed therapy. The median follow-up was 38 months. Multivariable analysis showed CVD history to be associated with a higher risk of overall mortality $(p=0.001)$. In the overall cohort and in patients without a history of CVD, patients who underwent local treatment exhibited higher OS than men who received ADT alone (all $p<0.001$ ). However, the survival benefit conferred by local treatment was not seen in patients with a history of CVD $(p=0.324)$. OS was comparable between patients who received RP and RT ( $p=0.521)$.

Conclusion: Local treatment with or without metastasis-directed therapy may provide OS advantages for mHSPC patients without a history of CVD. Further prospective studies are needed to address these important concerns.

Key Words: Cerebrovascular disorders, neoplasm metastasis, prostatic neoplasms, survival

\section{INTRODUCTION}

Advances in imaging techniques have led to the recognition of a transitional state of prostate cancer (PCa) in which the disease has limited metastasis beyond the prostate. In 1995, Hellman and Weichselbaum first identified and termed this entity as oligometastasis. ${ }^{1}$ Uppal, et al. ${ }^{2}$ suggested that metastatic disease between oligometastatic and polymetastatic patients have a separate regulation process, suggesting that the diseases mentioned above are distinct entities rather than different points

Received: August 16, 2019 Revised: September 9, 2019 Accepted: October 8, 2019

Corresponding author: Kyo Chul Koo, MD, PhD, Department of Urology, Gangnam Severance Hospital, Yonsei University College of Medicine, 211 Eonju-ro, Gangnam-gu, Seoul 06273, Korea.

Tel: 82-2-2019-3470, Fax: 82-2-3462-8887, E-mail: gckoo@yuhs.ac

-The authors have no potential conflicts of interest to disclose.

(C) Copyright: Yonsei University College of Medicine 2019

This is an Open Access article distributed under the terms of the Creative Commons Attribution Non-Commercial License (https://creativecommons.org/licenses/by-nc/4.0) which permits unrestricted non-commercial use, distribution, and reproduction in any medium, provided the original work is properly cited. 
along a development continuum. Other researchers indicated that definitive directed treatments for PCa with limited metastases may confer survival benefits for a select population. ${ }^{3}$

Androgen deprivation therapy (ADT) remains a cornerstone of systemic therapy through which to delay disease progression and to alleviate cancer-related symptoms in patients with metastatic hormone-sensitive prostate cancer (mHSPC). ${ }^{4}$ Many have regarded $\mathrm{ADT}$ as the treatment of choice for this population; however, recent evidence-based guidelines recommend concurrent aggressive treatment options, including local therapy or upfront chemotherapy. ${ }^{5}$ This recommendation is based on emerging data that suggest treatments targeted at the primary tumor and metastatic lesions in patients with limited metastases may prevent or delay the need for palliative treatments and confer survival benefits. ${ }^{6}$

The risks of cerebrovascular disease (CVD) and PCa increase with age, and $16 \%$ of patients with ischemic stroke have been found to harbor PCa. ${ }^{7}$ While an association between PCa and CVD has not been elucidated, cancers have been shown to be associated with an increased risk of CVD through various biological mechanisms, including direct tumor effects or coagulation disorders. ${ }^{8,9}$ Meanwhile, treatment decisions for PCa should be made based on a patient's comorbidities and functional status, which can affect overall survival (OS). Therefore, the risk of mortality from mHSPC and its treatment should be weighed carefully against the risk of mortality from existing significant comorbidities, such as CVD or cardiovascular disease, when making treatment decisions. ${ }^{10}$ Multiple comorbidities are frequently noted at the time of biopsy-proven mHSPC. Unfortunately, there is no consensus on which subgroup of patients for whom local treatment of the primary tumor may confer a survival benefit.

The primary endpoint of this study was the prognostic value of local treatment of primary tumors on OS outcomes in patients with de novo mHSPC. The secondary outcome was the confounding effect of comorbidities, such as CVD, at the time of mHSPC diagnosis on the prognostic impact of local treatment.

\section{MATERIALS AND METHODS}

\section{Study population and data collection}

Clinicopathological data for 1017 consecutive patients with newly diagnosed mHSPC between August 2003 and November 2016 were retrospectively collected from two institutions, Severance Hospital and Gangnam Severance Hospital (Yonsei University Health System). After stratifying patients by a history of CVD, baseline characteristics, including age, body mass index, prostate-specific antigen (PSA) level, TNM stage, Gleason score, Eastern Cooperative Oncology Group performance status, site of metastasis, preexisting medical conditions, such as hypertension and diabetes mellitus, and treatment types were reviewed. CVD was defined as previous radiological evidence of infarction on computed tomography brain scan identified by corresponding focal neurological deficit. Patients were excluded from the analysis if they had incomplete clinical data $(n=56)$, were lost during follow up $(\mathrm{n}=42)$, or had an unknown cause of death $(\mathrm{n}=40)$. In result, $879(86.4 \%)$ patients were included in the final analysis.

Patient survival and causes of death were investigated using the National Cancer Registry Database or institutional electronic medical records. The OS interval was defined as the time interval from PCa diagnosis to the date of all-cause death. Cancer-specific survival interval was defined as the time interval from PCa diagnosis to the date of death due to PCa. This retrospective study was approved by the Gangnam Severance Hospital Institutional Ethics Committee (2017-0186-001), which waived the requirement for informed consent. All study procedures complied with the principles of the 1946 Declaration of Helsinki and its 2008 update.

\section{Treatments administered}

Decisions on the type of initial treatment were determined based on physician discretion and patient preference. All patients received long-term $\mathrm{ADT}$ with or without local treatment [radical prostatectomy (RP) or radiation therapy (RT)]. RP was performed by a retropubic or robotic approach, with the extent of pelvic lymph node dissection based on risk category. RT consisted of intensity-modulated external beam RT, with a median RT dose of 7000 cGy (interquartile range: 7000-7000). Metastasisdirected local treatments, including RT and/or metastasectomy, were offered for patients with symptomatic disease or those with a limited metastatic burden.

The diagnosis of castration-resistant PCa (CRPC) was based on consecutive rises in serum PSA levels, new symptom development, or radiological progression despite a castrate serum testosterone level $<50 \mathrm{ng} / \mathrm{dL}$ during ADT. Systemic treatments targeted against CRPC were performed according to contemporary guidelines.

\section{Statistical analyses}

The study groups were compared using the two-sided MannWhitney U-test for the analysis of continuous variables and the chi-square test for the analysis of categorical variables. Variables considered potential predictors for multivariate modeling were selected by univariate analyses using the Cox proportional hazards regression model.

To compare the OS outcomes of each patient group, KaplanMeier survival analysis was performed according to prior CVD history and the type initial treatment (ADT alone vs. local treatment). All statistical analyses were performed using IBM SPSS software (version 21.0; IBM Corporation, Armonk, NY, USA). Differences with a $p$ value $<0.05$ were considered statistically significant. 


\section{RESULTS}

\section{Baseline characteristics}

Baseline features of the patients included in the analysis, stratified by prior CVD history and the type initial treatment, are described in Table 1. Of 879 patients, 660 (75.1\%) underwent ADT alone, 90 (10.2\%) underwent RT with ADT with or without metastasis-directed therapy, and 129 (14.7\%) underwent RP with ADT with or without metastasis-directed therapy.

Within all CVD history subgroups, patients who received ADT alone were older and exhibited higher Charlson Comorbidity Index (CCI) values than men who received local treatment. In the subgroup without a history of CVD, patients who received ADT alone had higher serum PSA levels than men who received local treatment. However, the two treatment subgroups were comparable in their distributions of all other classic PCa surviv- al prognosticators. Moreover, there was no significant difference in the number or sites of metastasis between the $\mathrm{ADT}$ alone and ADT with local treatment groups ( $p=0.521$, data not presented).

Systemic treatments administered following CRPC diagnosis are shown in Table 2. In the subgroup with CVD history, patients who received local treatment were more likely to have received a greater number of docetaxel cycles than men who received ADT alone. However, there were no differences in the proportions of androgen receptor axis-targeted agents, cabazitaxel usage, enrollment in clinical trials, and secondary hormonal manipulations.

\section{Survival outcomes}

Table 3 and Fig. 1 show the OS outcomes of the overall cohort and the subgroups stratified according to prior CVD history and the type of initial treatment. In the overall group (Fig. 1A) and

Table 1. Clinicopathological Characteristics of Patients with Metastatic Prostate Cancer Stratified by Cerebrovascular Disease History and Initial Treatment Category

\begin{tabular}{|c|c|c|c|c|c|c|c|}
\hline & & No CI & D history ( $n=802$ ) & & CVI & history (n=77) & \\
\hline & Overall ( $n=879$ ) & $\begin{array}{c}\text { ADT alone } \\
(\mathrm{n}=615)\end{array}$ & $\begin{array}{l}\text { Local treatment } \\
(n=187)\end{array}$ & $p$ value & $\begin{array}{c}\text { ADT alone } \\
(n=45)\end{array}$ & $\begin{array}{c}\text { Local treatment } \\
\quad(n=32)\end{array}$ & $p$ value \\
\hline Age & $66.5(61.0-71.8)$ & $70.0(65.0-76.0)$ & $67.0(62.0-73.0)$ & $<0.001$ & $74.0(69.0-78.8)$ & $68.0(63.3-72.8)$ & 0.001 \\
\hline Body mass index $\left(\mathrm{kg} / \mathrm{m}^{2}\right)$ & $23.5(21.5-25.2)$ & $23.7(21.8-25.6)$ & $23.9(21.8-25.5)$ & 0.293 & $23.8(22.2-26.3)$ & $23.6(21.4-25.6)$ & 0.525 \\
\hline PSA (ng/dL) & $69.2(15.0-182.0)$ & $123(55.8-466.1)$ & $37.6(14.1-142.8)$ & 0.004 & $123.7(35.0-396.3)$ & $63.2(17.3-261.3)$ & 0.359 \\
\hline Hypertension & $299(34.0)$ & $201(32.7)$ & $64(34.2)$ & 0.757 & $22(48.9)$ & $11(34.4)$ & 0.516 \\
\hline Diabetes mellitus & $151(17.2)$ & $104(16.9)$ & $27(14.4)$ & 0.565 & $13(28.9)$ & $5(15.6)$ & 0.485 \\
\hline Liver cirrhosis & $14(1.6)$ & $9(1.5)$ & $2(1.1)$ & 0.545 & $2(4.4)$ & $1(3.1)$ & 0.468 \\
\hline T stage & & & & & & & 0.246 \\
\hline$\leq \mathrm{T} 2$ & $124(14.1)$ & $85(13.8)$ & $24(12.8)$ & & $12(26.7)$ & 4 (12.5) & \\
\hline$\geq \mathrm{T} 3$ & $755(85.9)$ & $530(86.2)$ & $163(87.2)$ & & $33(73.3)$ & $28(87.5)$ & \\
\hline $\mathrm{N}$ stage & & & & 0.495 & & & 0.145 \\
\hline NO & $356(40.5)$ & $239(38.9)$ & $78(41.7)$ & & $24(53.3)$ & $15(46.9)$ & \\
\hline N1 & $523(59.5)$ & $376(61.1)$ & $109(58.3)$ & & $21(46.7)$ & $17(53.1)$ & \\
\hline Site of metastasis & & & & & & & \\
\hline Bone & $605(68.8)$ & 431 (70.1) & $123(65.8)$ & 0.154 & $31(68.9)$ & $20(62.5)$ & 0.485 \\
\hline Visceral & $35(4.0)$ & $31(5.0)$ & $2(1.1)$ & 0.864 & $2(4.4)$ & $0(0.0)$ & 0.138 \\
\hline Lymph node & $423(48.1)$ & $315(51.2)$ & $70(37.4)$ & 0.132 & $24(53.3)$ & $14(43.8)$ & 0.468 \\
\hline Number of metastasis & & & & 0.546 & & & 0.588 \\
\hline$\leq 5$ & $671(76.3)$ & $485(78.9)$ & $129(69.0)$ & & $34(75.6)$ & $23(71.9)$ & \\
\hline$>5$ & $208(23.7)$ & $130(21.1)$ & $58(31.0)$ & & $11(24.4)$ & $9(28.1)$ & \\
\hline Gleason score & & & & 0.051 & & & 0.145 \\
\hline$\leq 7$ & $174(19.8)$ & $103(16.7)$ & $43(23.0)$ & & $18(40.0)$ & $10(31.2)$ & \\
\hline$\geq 8$ & $705(80.2)$ & $512(83.3)$ & $144(77.0)$ & & $27(60.0)$ & $22(68.8)$ & \\
\hline $\mathrm{CCl}$ & & & & 0.039 & & & 0.017 \\
\hline$\leq 1$ & $577(65.6)$ & $429(69.8)$ & $115(61.5)$ & & $12(26.7)$ & $21(65.6)$ & \\
\hline$\geq 2$ & $302(34.4)$ & $186(30.2)$ & 72 (38.5) & & $33(73.3)$ & $11(34.4)$ & \\
\hline ECOG PS & & & & $<0.001$ & & & 0.359 \\
\hline 0 & $778(88.5)$ & $568(92.4)$ & $146(78.1)$ & & 39 (86.7) & $25(78.1)$ & \\
\hline$\geq 1$ & $101(11.5)$ & $47(7.6)$ & $41(21.9)$ & & $6(13.3)$ & 7 (21.9) & \\
\hline
\end{tabular}

CVD, cerebrovascular disease; ADT, androgen-deprivation therapy; PSA, prostate-specific antigen; CCI, Charlson Comorbidity Index; ECOG PS, Eastern Cooperative Oncology Group performance status.

Data are presented as medians (interquartile range) and numbers (\%). 
in patients without a history of CVD (Fig. 1B), men who received local treatment exhibited higher OS than men who received ADT alone (all $p<0.001)$. However, the survival benefit of local treatment was not seen in the subgroup of patients with a history of CVD ( $p=0.324)$ (Fig. 1C). OS outcomes were comparable between patients who received RP and RT ( $p=0.521$, data not presented). The leading causes of death were attributable to PCa, followed by second primary malignancy and cardiovascular disease (Table 3 ).

\section{Predictors of survival}

Univariate and multivariate models of predictors of overall mortality (OM) are shown in Tables 4, 5, and 6. In the overall group and the subgroup without a history of CVD, multivariate analysis showed local treatment to be associated with a lower risk of OM, whereas this was not seen in the subgroup with a history of CVD.

In the overall group, serum PSA level, history of CVD, stage $\geq \mathrm{T} 3$, and Gleason score $\geq 8$ were significant prognosticators of

Table 2. Systemic Treatments Administered Following Progression to Castration-Resistant Prostate Cancer

\begin{tabular}{|c|c|c|c|c|c|c|c|}
\hline & \multirow[b]{2}{*}{ Overall ( $n=879$ ) } & \multicolumn{3}{|c|}{ No CVD history ( $n=802$ ) } & \multicolumn{3}{|c|}{ CVD history (n=77) } \\
\hline & & $\begin{array}{c}\text { ADT alone } \\
(n=615)\end{array}$ & $\begin{array}{l}\text { Local treatment } \\
\qquad(\mathrm{n}=187)\end{array}$ & $p$ value & $\begin{array}{c}\text { ADT alone } \\
(n=45)\end{array}$ & $\begin{array}{l}\text { Local treatment } \\
\qquad(\mathrm{n}=32)\end{array}$ & $p$ value \\
\hline \multicolumn{8}{|l|}{ Docetaxel } \\
\hline $\mathrm{N}$ & $214(24.3)$ & $151(24.6)$ & 34 (18.2) & 0.155 & $10(22.2)$ & 19 (59.4) & 0.001 \\
\hline No. cycles & $4.0(2.0-9.0)$ & $5.0(3.0-8.5)$ & $3.0(8.5-12.5)$ & 0.484 & $4.0(2.0-11.5)$ & $5.0(4.0-7.5)$ & 0.587 \\
\hline \multicolumn{8}{|l|}{ ARAT agent use } \\
\hline Pre-chemotherapy & $33(3.8)$ & $23(3.7)$ & $7(3.7)$ & 0.654 & $1(2.2)$ & $2(6.3)$ & 0.456 \\
\hline Abiraterone & $12(1.4)$ & $8(1.3)$ & $2(1.1)$ & 0.819 & $0(0.0)$ & $2(6.3)$ & 0.170 \\
\hline Enzalutamide & $21(2.4)$ & $15(2.4)$ & $5(2.7)$ & 0.801 & $1(2.2)$ & $0(0.0)$ & 0.912 \\
\hline Post-chemotherapy & $101(11.5)$ & $55(8.9)$ & $29(15.5)$ & 0.644 & $5(11.1)$ & $12(37.5)$ & 0.564 \\
\hline Abiraterone & $27(3.1)$ & $13(2.1)$ & $9(4.8)$ & 0.104 & $1(2.2)$ & $4(12.5)$ & 0.079 \\
\hline Enzalutamide & $74(8.4)$ & $42(6.8)$ & $20(10.7)$ & 0.207 & $4(8.9)$ & $8(25.0)$ & 0.065 \\
\hline Cabazitaxel & $8(0.9)$ & $6(1.0)$ & $2(1.1)$ & 0.954 & $0(0.0)$ & $0(0.0)$ & 0.556 \\
\hline Clinical trials & $71(8.1)$ & $59(9.6)$ & $7(3.7)$ & 0.067 & $4(8.9)$ & $1(3.1)$ & 0.312 \\
\hline $\begin{array}{l}\text { Secondary hormonal } \\
\text { manipulation }\end{array}$ & $12(1.4)$ & $8(1.3)$ & $2(1.1)$ & 0.819 & $0(0.0)$ & $2(6.3)$ & 0.089 \\
\hline
\end{tabular}

CVD, cerebrovascular disease; ADT, androgen-deprivation therapy; ARAT, androgen receptor-axis targeted.

Data are presented as medians (interquartile range) and numbers (\%).

Table 3. Survival Outcomes in Patients with Metastatic Prostate Cancer Stratified according to Cerebrovascular Disease History and Initial Treatment Category

\begin{tabular}{|c|c|c|c|c|c|c|c|}
\hline & \multirow[b]{2}{*}{ Overall $(n=879)$} & \multicolumn{3}{|c|}{ No CVD history (n=802) } & \multicolumn{3}{|c|}{ CVD history (n=77) } \\
\hline & & $\begin{array}{l}\text { ADT alone } \\
(n=615)\end{array}$ & $\begin{array}{l}\text { Local treatment } \\
\quad(n=187)\end{array}$ & $p$ value & $\begin{array}{c}\text { ADT alone } \\
(n=45)\end{array}$ & $\begin{array}{l}\text { Local treatment } \\
\qquad(n=32)\end{array}$ & $p$ value \\
\hline OS, 5-year (\%) & 45.9 & 44.4 & 68.1 & $<0.001$ & 32.1 & 39.1 & 0.324 \\
\hline No. all-cause deaths (n, \%) & $577(65.6)$ & $446(72.5)$ & $103(55.1)$ & $<0.001$ & $32(71.1)$ & 23 (71.9) & 0.845 \\
\hline \multicolumn{8}{|l|}{ Cause of death $(n, \%)$} \\
\hline $\mathrm{PCa}$ & $493(56.1)$ & $370(60.2)$ & $71(38.0)$ & $<0.001$ & $30(66.7)$ & $22(68.8)$ & 0.847 \\
\hline Second primary malignancy & $58(6.6)$ & $41(6.7)$ & $15(8.0)$ & 0.468 & $1(2.2)$ & $1(3.1)$ & 0.344 \\
\hline Cardiovascular disease & $15(1.7)$ & $10(1.6)$ & $5(2.7)$ & 0.168 & $0(0.0)$ & $0(0.0)$ & NA \\
\hline Cerebrovascular disease & $4(0.5)$ & $2(0.3)$ & $2(1.1)$ & 0.453 & $0(0.0)$ & $0(0.0)$ & NA \\
\hline Liver cirrhosis & $3(0.3)$ & $1(0.2)$ & $2(1.1)$ & 0.135 & $0(0.0)$ & $0(0.0)$ & NA \\
\hline Pneumonia & $6(0.6)$ & $6(1.0)$ & $0(0.0)$ & 0.642 & $0(0.0)$ & $0(0.0)$ & NA \\
\hline Renal disease & $5(0.6)$ & $3(0.5)$ & $1(0.5)$ & 0.445 & $1(2.2)$ & $0(0.0)$ & 0.264 \\
\hline Self-harm & $1(0.1)$ & $1(0.2)$ & $0(0.0)$ & 0.474 & $0(0.0)$ & $0(0.0)$ & NA \\
\hline Other & $19(2.2)$ & $12(2.0)$ & $7(3.7)$ & 0.344 & $0(0.0)$ & $0(0.0)$ & NA \\
\hline Median OS period (mon) & $38.0(23.2-64.0)$ & $34.0(10.0-59.3)$ & $34.0(22.0-67.3)$ & 0.512 & $30.0(21.5-45.0)$ & $36.0(24.0-56.0)$ & 0.565 \\
\hline Follow-up period (mon) & $45.0(26.0-76.0)$ & $43.0(24.0-73.0)$ & $53.0(28.0-75.0)$ & 0.655 & $31.5(20.0-58.5)$ & $40.5(26.3-60.8)$ & 0.131 \\
\hline
\end{tabular}

CVD, cerebrovascular disease; ADT, androgen-deprivation therapy; OS, overall survival; PCa, prostate cancer; NA, not available.

Data are presented as numbers (\%) and medians (interquartile range). 
OM (Table 4). In the subgroup without a history of CVD, stage $\geq \mathrm{T} 3$ and Gleason score $\geq 8$ were associated with a higher risk of $\mathrm{OM}$ (Table 5). In the subgroup with a history of CVD, serum PSA level and CCI were associated with a higher risk of OM (Table 6).

Univariate and multivariate models of predictors for cancerspecific mortality in the overall group are shown in Table 7. Age, stage $\geq \mathrm{T} 3$, Gleason score $\geq 8$, and prior local treatment were significant prognosticators (Table 7 ).

\section{DISCUSSION}

There has been a paradigm shift in considering local treatments targeted at both the primary tumor and metastatic lesions in patients with mHSPC and limited metastatic burden. ${ }^{6}$ However, there is no guideline on which subgroup of patients for whom such aggressive treatments may confer a survival benefit. We observed an OS benefit with local treatment of the primary tumor in patients regardless of the metastatic burden. However, the survival benefit with local treatment was not seen in patients with a prior history of CVD. These findings suggest that comorbidities are crucial factors that affect the survival benefit of lo-

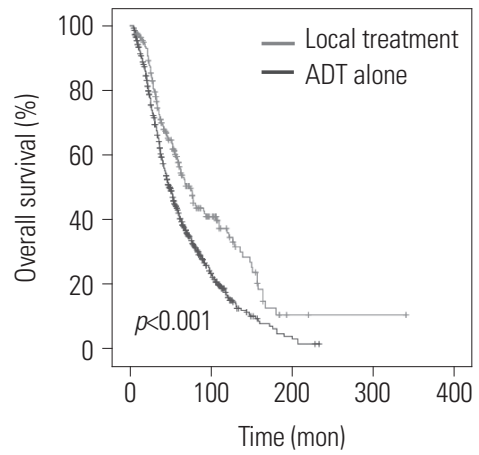

Number at risk

Local treatment $219 \quad 47 \quad 2 \quad 1 \quad 0$ ADT alone $\begin{array}{lllll}660 & 84 & 4 & 0 & 0\end{array}$

A

Fig. 1. Kaplan-Meier curves showing overall survival in patients stratified by a history of cerebrovascular disease (CVD). (A) Overall group, (B) patients without a history of CVD, and (C) patients with a history of CVD. ADT, androgen-deprivation therapy.

Table 4. Cox Regression Models for Risk Factors associated with Overall Mortality in the Overall Cohort

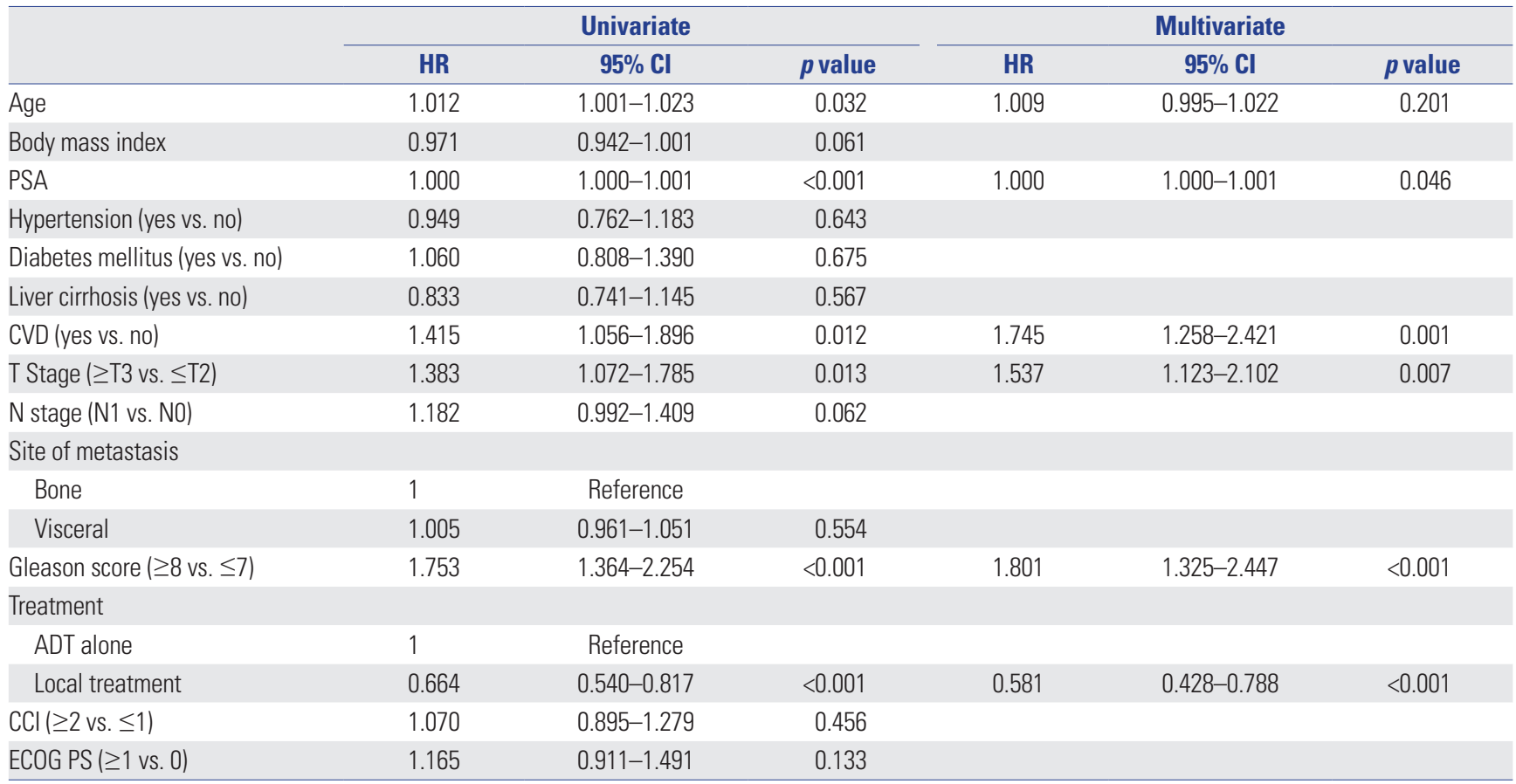

$\mathrm{HR}$, hazards ratio; $\mathrm{Cl}$, confidence interval; PSA, prostate-specific antigen; CVD, cerebrovascular disease; ADT, androgen-deprivation therapy; CCI, Charlson comorbidity score; ECOG PS, Eastern Cooperative Oncology Group performance status. 
cal treatment in patients with mHSPC.

The biological definition of oligometastatic PCa remains debatable, and advances in imaging techniques are shifting the treatment paradigm of this disease entity. Various studies have proposed different definitions regarding the number and sites of metastatic lesions to define oligometastatic PCa based on oncological outcomes. ${ }^{11,12}$ The definition regarding the cut-off number of metastatic lesions is yet controversial, and for now, this disease entity should only be interpreted as a disease state between the presence of intravascular circulating tumor cells and disseminated metastasis. ${ }^{13,14}$ Existing studies regarding oligometastatic PCa exclude patients with underlying comorbidities, such as CVD. ${ }^{15-19}$ Our study cohort included patients with initially diagnosed mHSPC regardless of the number of meta-

Table 5. Cox Regression Models for Risk Factors associated with Overall Mortality in Patients without a History of Cerebrovascular Disease

\begin{tabular}{|c|c|c|c|c|c|c|}
\hline & & Univariate & & & Multivariate & \\
\hline & HR & $95 \% \mathrm{CI}$ & $p$ value & HR & $95 \% \mathrm{Cl}$ & $p$ value \\
\hline Age & 1.009 & $0.997-1.020$ & 0.130 & & & \\
\hline Body mass index & 0.975 & $0.944-1.007$ & 0.129 & & & \\
\hline PSA & 1.000 & $1.000-1.001$ & 0.013 & 1.000 & $1.000-1.001$ & 0.271 \\
\hline Hypertension (yes vs. no) & 0.905 & $0.717-1.142$ & 0.399 & & & \\
\hline Diabetes mellitus (yes vs. no) & 1.001 & $0.746-1.344$ & 0.992 & & & \\
\hline Liver cirrhosis (yes vs. no) & 1.083 & $0.908-1.305$ & 0.183 & & & \\
\hline T Stage ( $\geq T 3$ vs. $\leq \mathrm{T} 2$ ) & 1.368 & $1.044-1.791$ & 0.023 & 1.461 & $1.101-1.939$ & 0.009 \\
\hline N stage (N1 vs. N0) & 1.156 & $0.962-1.390$ & 0.123 & & & \\
\hline Site of metastasis & & & & & & \\
\hline Bone & 1 & Reference & & & & \\
\hline Visceral & 1.103 & $0.971-1.225$ & 0.485 & & & \\
\hline Gleason score ( $\geq 8$ vs. $\leq 7$ ) & 1.773 & $1.356-2.318$ & $<0.001$ & 1.635 & $1.243-2.151$ & $<0.001$ \\
\hline Treatment & & & & & & \\
\hline ADT alone & 1 & Reference & & & & \\
\hline Local treatment & 0.626 & $0.499-0.786$ & $<0.001$ & 0.630 & $0.495-0.800$ & $<0.001$ \\
\hline $\mathrm{CCl}(\geq 2$ vs. $\leq 1)$ & 0.982 & $0.811-1.191$ & 0.857 & & & \\
\hline ECOG PS ( $\geq 1$ vs. 0) & 1.176 & $0.903-1.531$ & 0.231 & & & \\
\hline
\end{tabular}

$\mathrm{HR}$, hazards ratio; $\mathrm{Cl}$, confidence interval; PSA, prostate-specific antigen; ADT, androgen-deprivation therapy; CCI, Charlson comorbidity score; ECOG PS, Eastern Cooperative Oncology Group performance status.

Table 6. Cox Regression Models for Risk Factors associated with Overall Mortality in Patients with a History of Cerebrovascular Disease

\begin{tabular}{|c|c|c|c|c|c|c|}
\hline & \multicolumn{3}{|c|}{ Univariate } & \multicolumn{3}{|c|}{ Multivariate } \\
\hline & HR & $95 \% \mathrm{Cl}$ & $p$ value & HR & $95 \% \mathrm{Cl}$ & $p$ value \\
\hline Age & 1.038 & $1.001-1.075$ & 0.044 & 1.026 & $0.989-1.064$ & 0.174 \\
\hline Body mass index & 0.933 & $0.842-1.034$ & 0.184 & & & \\
\hline PSA & 1.000 & $1.000-1.001$ & 0.007 & 1.000 & $1.000-1.001$ & 0.029 \\
\hline Hypertension (yes vs. no) & 1.447 & $0.723-2.897$ & 0.297 & & & \\
\hline Diabetes mellitus (yes vs. no) & 1.383 & $0.653-2.928$ & 0.397 & & & \\
\hline Liver cirrhosis (yes vs. no) & 1.106 & $0.776-1.486$ & 0.329 & & & \\
\hline T Stage ( $\geq T 3$ vs. $\leq T 2$ ) & 1.699 & $0.761-3.789$ & 0.196 & & & \\
\hline N stage (N1 vs. N0) & 1.468 & $0.804-2.678$ & 0.211 & & & \\
\hline \multicolumn{7}{|l|}{ Site of metastasis } \\
\hline Bone & 1 & Reference & & & & \\
\hline Visceral & 0.831 & $0.723-1.451$ & 0.468 & & & \\
\hline Gleason score ( $\geq 8$ vs. $\leq 7)$ & 1.869 & $0.900-3.884$ & 0.094 & & & \\
\hline \multicolumn{7}{|l|}{ Treatment } \\
\hline ADT alone & 1 & Reference & & & & \\
\hline Local treatment & 0.772 & $0.441-1.360$ & 0.364 & & & \\
\hline $\mathrm{CCl}(\geq 2$ vs. $\leq 1)$ & 2.006 & $1.099-3.663$ & 0.023 & 2.024 & $1.075-3.910$ & 0.029 \\
\hline ECOG PS ( $\geq 1$ vs. 0) & 1.101 & $0.547-2.213$ & 0.788 & & & \\
\hline
\end{tabular}

$\mathrm{HR}$, hazards ratio; $\mathrm{Cl}$, confidence interval; PSA, prostate-specific antigen; ADT, androgen-deprivation therapy; CCI, Charlson comorbidity score; ECOG PS, Eastern Cooperative Oncology Group performance status. 
Table 7. Cox Regression Models for Risk Factors associated with Cancer-Specific Mortality in the Overall Cohort

\begin{tabular}{|c|c|c|c|c|c|c|}
\hline & \multicolumn{3}{|c|}{ Univariate } & \multicolumn{3}{|c|}{ Multivariate } \\
\hline & HR & $95 \% \mathrm{Cl}$ & $p$ value & HR & $95 \% \mathrm{CI}$ & $p$ value \\
\hline Age & 1.020 & $1.010-1.031$ & $<0.001$ & 1.015 & $1.003-1.027$ & 0.016 \\
\hline Body mass index & 0.964 & $0.937-0.992$ & 0.013 & 0.976 & $0.946-1.006$ & 0.110 \\
\hline PSA & 1.000 & $1.000-1.001$ & $<0.001$ & 1.000 & $1.000-1.001$ & 0.070 \\
\hline Hypertension (yes vs. no) & 1.012 & $0.827-1.239$ & 0.906 & & & \\
\hline Diabetes mellitus (yes vs. no) & 1.171 & $0.917-1.496$ & 0.205 & & & \\
\hline Liver cirrhosis (yes vs. no) & 0.731 & $0.539-1.010$ & 0.554 & & & \\
\hline CVD (yes vs. no) & 1.275 & $0.961-1.691$ & 0.093 & & & \\
\hline $\mathrm{T}$ Stage ( $\geq \mathrm{T} 3$ vs. $\leq \mathrm{T} 2$ ) & 1.281 & $1.014-1.618$ & 0.038 & 1.417 & $1.076-1.864$ & 0.013 \\
\hline N stage (N1 vs. N0) & 1.109 & $0.941-1.307$ & 0.216 & & & \\
\hline \multicolumn{7}{|l|}{ Site of metastasis } \\
\hline Bone & 1 & Reference & & & & \\
\hline Visceral & 1.093 & $0.912-1.321$ & 0.564 & & & \\
\hline Gleason score ( $\geq 8$ vs. $\leq 7$ ) & 1.462 & $1.172-1.824$ & 0.001 & 1.437 & $1.125-1.835$ & 0.004 \\
\hline \multicolumn{7}{|l|}{ Treatment } \\
\hline ADT alone & 1 & Reference & & & & \\
\hline Local treatment & 0.635 & $0.521-0.774$ & $<0.001$ & 0.677 & $0.547-0.838$ & $<0.001$ \\
\hline $\mathrm{CCl}(\geq 2$ vs. $\leq 1)$ & 1.180 & 0.999-1.393 & 0.051 & & & \\
\hline ECOG PS ( $\geq 1$ vs. 0) & 1.055 & $0.832-1.341$ & 0.663 & & & \\
\hline
\end{tabular}

$\mathrm{HR}$, hazards ratio; $\mathrm{Cl}$, confidence interval; PSA, prostate-specific antigen; CVD, cerebrovascular disease; ADT, androgen-deprivation therapy; CCI, Charlson comorbidity score; ECOG PS, Eastern Cooperative Oncology Group performance status.

static lesions and comorbidities, and there was no significant difference in metastatic burden between the ADT alone and ADT with local treatment groups. In an attempt to identify a subset of patients who would benefit from local treatment of $\mathrm{mH}-$ SPC, our study suggests that the number of metastatic lesions may not be the most significant indicator for predicting a survival benefit with local treatment.

Our study showed that CVD, followed by Gleason score, was the strongest prognosticator of OS. Several hypotheses can be offered. First, the detrimental effect of CVD on OS may have offset the beneficial effect of local treatment, in addition to wellknown prognostic factors, such as Gleason score and tumor stage. CVD is known to be significantly associated with surviv$\mathrm{al}$, with a 0.15-year loss of life expectancy in the aged population. ${ }^{20}$ It has been reported that the risk of thromboembolic disease increases as cancer stage increases. ${ }^{21}$ Babiker, et al. ${ }^{22}$ showed that the early release of prostasomes originating from PCa cells into the bloodstream evokes coagulation effects resulting in an increased risk of thromboembolism. Brain injury resulting from vascular pathology can also activate the coagulation cascade, causing a state of hypercoagulability with the release of thromboplastin-like factors from the central nervous system. ${ }^{23}$ In summary, a history of CVD, which indicates pathological hemostatic changes, may contribute to the disease course and OS outcomes. Future studies are warranted to identify the underlying pathophysiology of CVD in the PCa microenvironment. Second, the administration of continuous ADT may exacerbate CVD and its disease course. ADT increases coagulation disorders that are related to a complex interplay of factors, including procoagulant factors released by tumor and/or host blood vessels. ${ }^{22}$ Hypercoagulability is common in cancer patients, and hence, it is reasonable to consider that ADT serves as an aggravator of CVD and inferior OS outcomes. Our results imply that comorbidities, especially CVD, are potential risk factors to be accounted for when considering local treatments in addition to ADT for patients with mHSPC.

The strengths of our study are the inclusion of detailed clinicopathologic data, treatment information, comorbidities, and performance status that were available from each patient. At the same time, some limitations exist. First, due to the observational nature of the study, the results should only be interpreted within the limitation of a retrospective design. Diagnosis and ascertainment of CVD were dependent on chart reviews. While it is likely that there are undiagnosed cases, the use of medical records is a well-established method to consistently identify CVD outcomes within structured data. Second, the heterogeneity of the study cohort and the lack of a standard therapeutic approach, due to the strong patient and physician preference regarding the implementation of specific treatment modalities, were also limitations of this study. Nevertheless, we believe that such bias would be inherent in any retrospective study and may reflect the reality of clinical practice in which the application of sequential therapies for mHSPC is not standardized. Third, in the majority ( $87.3 \%$ ) of patients, the presence of metastasis was determined from imaging studies and was not pathologically confirmed. Therefore, a proportion of our cohort might have harbored non-metastatic disease. Fourth, the possibility of unidentified imbalance in baseline patients and tumor character- 
istics cannot be overlooked, along with the absence of a formal follow-up protocol, which may potentially confound the results of this study. Lastly, while cancer-specific survival may be considered as the most robust survival endpoint, we chose to use OS as the endpoint, since our study population included patients who were relatively older in age and had multiple comorbidities, in which $34 \%$ of the patients had CCI values $\geq 2$. Indeed, as OS is affected by competing risks, we considered that OS may reliably reflect the impact of CVD on survival in real-life cancer care.

A variety of factors influence outcomes in patient with CVD in the setting of underlying cancer. Local treatment with or without metastasis-directed therapy may provide an OS advantage for de novo mHSPC patients without a history of CVD. While this association requires confirmation in a prospectively designed study, patients with PCa with a prior history of CVD may be offered less aggressive treatment options.

\section{ACKNOWLEDGEMENTS}

This study was supported by the Young Researcher Program Grant of the National Research Foundation of Korea (NRF2017R1C1B5017516)

\section{AUTHOR CONTRIBUTIONS}

Conceptualization: Tae Jin Kim and Kyo Chul Koo. Data curation: Koon Ho Rha, Sung Joon Hong, Byung Ha Chung, and Kyo Chul Koo. Formal analysis: Tae Jin Kim and Kyo Chul Koo. Funding acquisition: Byung Ha Chung and Kyo Chul Koo. Investigation: Young Dong Yu and Dong Soo Park. Methodology: Tae Jin Kim and Young Dong Yu. Project administration: Koon Ho Rha, Sung Joon Hong, and Kang Su Cho. Resources: Byung Ha Chung and Kyo Chul Koo. Software: Young Dong Yu. Supervision: Dong Soo Park and Byung Ha Chung. Validation: Kyo Chul Koo, Dong Soo Park, and Byung Ha Chung. Visualization: Koon Ho Rha and Sung Joon Hong. Writing_original draft: Tae Jin Kim and Kyo Chul Koo. Writing — review \& editing: Tae Jin Kim and Kyo Chul Koo.

\section{ORCID iDs}

Tae Jin Kim Young Dong Yu Dong Soo Park Koon Ho Rha Sung Joon Hong Kang Su Cho Byung Ha Chung Kyo Chul Koo https://orcid.org/0000-0001-7836-1998 https://orcid.org/0000-0002-2696-7004 https://orcid.org/0000-0002-1377-3630 https://orcid.org/0000-0001-8588-7584 https://orcid.org/0000-0001-9869-065X https://orcid.org/0000-0002-3500-8833 https://orcid.org/0000-0001-9817-3660 https://orcid.org/0000-0001-7303-6256

\section{REFERENCES}

1. Hellman S, Weichselbaum RR. Oligometastases. J Clin Oncol 1995;13:8-10.

2. Uppal A, Ferguson MK, Posner MC, Hellman S, Khodarev NN, Weichselbaum RR. Towards a molecular basis of oligometastatic disease: potential role of micro-RNAs. Clin Exp Metastasis 2014; 31:735-48.
3. Park JW, Jang WS, Koh DH, Ham WS, Rha KH, Hong SJ, et al. Impact of early salvage androgen deprivation therapy in localized prostate cancer after radical prostatectomy: a propensity score matched analysis. Yonsei Med J 2018;59:580-7.

4. Sharifi N, Gulley JL, Dahut WL. Androgen deprivation therapy for prostate cancer. JAMA 2005;294:238-44.

5. Mohler JL, Antonarakis ES, Armstrong AJ, D’Amico AV, Davis BJ, Dorff T, et al. Prostate Cancer, Version 2.2019, NCCN Clinical Practice Guidelines in oncology. J Natl Compr Canc Netw 2019;17: 479-505.

6. Koo KC, Dasgupta P. Treatment of oligometastatic hormone-sensitive prostate cancer: a comprehensive review. Yonsei Med J 2018;59:567-79.

7. Selvik HA, Thomassen L, Logallo N, Næss H. Prior cancer in patients with ischemic stroke: the Bergen NORSTROKE study. J Stroke Cerebrovasc Dis 2014;23:919-25.

8. Grisold W, Oberndorfer S, Struhal W. Stroke and cancer: a review. Acta Neurol Scand 2009;119:1-16.

9. Levine SR. Hypercoagulable states and stroke: a selective review. CNS Spectr 2005;10:567-78.

10. Crawford ED, Higano CS, Shore ND, Hussain M, Petrylak DP. Treating patients with metastatic castration resistant prostate cancer: a comprehensive review of available therapies. J Urol 2015; 194:1537-47.

11. Bolla M, Collette L, Blank L, Warde P, Dubois JB, Mirimanoff RO, et al. Long-term results with immediate androgen suppression and external irradiation in patients with locally advanced prostate cancer (an EORTC study): a phase III randomized trial. Lancet 2002; 360:103-6.

12. Strong VE, D’Angelica M, Tang L, Prete F, Gönen M, Coit D, et al. Laparoscopic adrenalectomy for isolated adrenal metastasis. Ann Surg Oncol 2007;14:3392-400.

13. Aoun F, Peltier A, van Velthoven R. A comprehensive review of contemporary role of local treatment of the primary tumor and/or the metastases in metastatic prostate cancer. Biomed Res Int 2014;2014:501213.

14. Tosoian JJ, Gorin MA, Ross AE, Pienta KJ, Tran PT, Schaeffer EM. Oligometastatic prostate cancer: definitions, clinical outcomes and treatment considerations. Nat Rev Urol 2017;14:15-25.

15. Keating NL, O'Malley A, Freedland SJ, Smith MR. Diabetes and cardiovascular disease during androgen deprivation therapy: observational study of veterans with prostate cancer. J Natl Cancer Inst 2012;104:1518-23.

16. Morgans AK, Fan KH, Koyama T, Albertsen PC, Goodman M, Hamilton AS, et al. Bone complications among prostate cancer survivors: long-term follow-up from the prostate cancer outcomes study. Prostate Cancer Prostatic Dis 2014;17:338-42.

17. Saigal CS, Gore JL, Krupski TL, Hanley J, Schonlau M, Litwin MS, et al. Androgen deprivation therapy increases cardiovascular morbidity in men with prostate cancer. Cancer 2007;110:1493-500.

18. Tsai HK, D'Amico AV, Sadetsky N, Chen MH, Carroll PR. Androgen deprivation therapy for localized prostate cancer and the risk of cardiovascular mortality. J Natl Cancer Inst 2007;99:1516-24.

19. Jespersen CG, Nørgaard M, Borre M. Androgen-deprivation therapy in treatment of prostate cancer and risk of myocardial infarction and stroke: a nationwide Danish population-based cohort study. Eur Urol 2014;65:704-9.

20. Li GQ, Fan J, Liu J, Wang W, Wang M, Qi Y, et al. Impact of cerebrovascular disease mortality on life expectancy in China. Biomed Environ Sci 2014;27:169-75.

21. Nanda A, Chen MH, Braccioforte MH, Moran BJ, D'Amico AV. Hormonal therapy use for prostate cancer and mortality in men with coronary artery disease-induced congestive heart failure or myo- 
Tae Jin Kim, et al.

cardial infarction. JAMA 2009;302:866-73.

22. Babiker AA, Ekdahl KN, Nilsson B, Ronquist G. Prothrombotic effects of prostasomes isolated from prostatic cancer cell lines and seminal plasma. Semin Thromb Hemost 2007;33:80-6.
23. Kaur H, Siemens DR, Black A, Robb S, Barr S, Graham CH, et al. Effects of androgen-deprivation therapy on hypercoagulability in prostate cancer patients: a prospective, longitudinal study. Can Urol Assoc J 2017;11:33-8. 